American Journal of Applied Sciences 5 (2): 165-168, 2008

ISSN 1546-9239

(C) 2008 Science Publications

\title{
Thermal Diffusivity of Carbon Pellets (CPs) Treated with KOH
}

\author{
${ }^{1} \mathrm{M}$ Haydari, ${ }^{2} \mathrm{M}$ M Moksin, ${ }^{3} \mathrm{~A}$ E Abdelrahman, ${ }^{3} \mathrm{M}$ Deraman, ${ }^{2} \mathrm{~W}$ M M Yunus, and ${ }^{2} \mathrm{I}$ V Grozescu \\ Department of physics, Faculty of Science, Shahed University, 3319118651, Tehran. Iran \\ ${ }^{2}$ Department of physics, Faculty of Science, Universiti Putra Malaysia, 43400 UPM Serdang. Malaysia \\ ${ }^{3}$ School of Applied Physics, Faculty of Science and Technology, Universiti Kebangsaan Malaysia, 43600 \\ UKM Bangi. Malaysia
}

\begin{abstract}
In this work thermal diffusivity of carbon pellets (CPs) treated with different percentage level of $\mathrm{KOH}$ has been studied. Thermal diffusivity measurements were carried out at room temperature by using photoflash technique. The technique consists of a camera flash having approximately $5 \mathrm{~ms}$ pulse duration for heating and a thin film of polyvinylidene difluoride (PVDF) attached to the back of the samples for signal detection. Eight carbon samples treated with different percentage level of $\mathrm{KOH}$ ( 0 to 7 mole percent) were studied and the thermal diffusivity result was compared with SEM which showed that the sample with highest thermal diffusivity had distinctly lower porosity and better grain alignment.
\end{abstract}

Keyword : Thermal Diffusivty, KOH, PVDF, Flash Technique

\section{INTRODUCTION}

The porosity effect on thermal properties of material has been studied by many workers since $1972^{[1,2,3]}$. Since that time a lot of work has been done on porosity effect on thermal conductivity of materials. In comparison, there was less work done on porosity effect on thermal diffusivity ${ }^{[4,5]}$. Beside porosity, the grain size and grain alignment in microstructure of materials are two other important parameters that can affect thermal properties of materials as has been reported by Haydari et al ${ }^{[6]}$. In this work the effect of porosity and grain alignment on thermal diffusivity was studied on the samples of carbon pellets (CPs) treated with $\mathrm{KOH}$. Photoflash technique was used to measure thermal diffusivity whereby camera flash absorption at the front surface induced heat into the sample while the temperature history of the rear surface of the sample was detected by using polyvinylidene difluoride (PVDF) film.

\section{MATERIAL AND METHODS}

Theory: One-dimensional heat propagation in a system consisting of four layers of gas, sample, PVDF sensor and backing, assuming that the sample is optically opaque and the backing has similar thermal properties to the PVDF sensor was considered. The theoretical calculation of the signal as detected by PVDF sensor that thermally attached to the sample was obtained by approximating the long camera flash temporal profile with a finite square pulse ${ }^{[7]}$.

Sample preparation: Carbon pellets (CPs) precursor (cotton cellulose) was first pre-carbonized at low carbonization temperature, milled for $20 \mathrm{~h}$ and sieved to produce self-adhesive carbon grains (SACG) that can pass through a 53 microns sieve. The SACG was treated for 16 hours with $\mathrm{KOH}$ having concentration from 0 to 7 moles percent and then dried in oven at 100 ${ }^{\circ} \mathrm{C}$ before converted into pellets (CPs) by applying 15 metric tones compression pressure on $2 \mathrm{~g}$ of samples in a mould. These CPs were carbonized from $500{ }^{\circ} \mathrm{C}$ to $1000{ }^{\circ} \mathrm{C}$ in nitrogen environment using a multi-steps heating profile (Vulcan Box Furnace 3-1750). The CPs were then washed with distilled water to remove impurities until a pH of 6 and dried in an oven at 100 ${ }^{\circ} \mathrm{C}$ for 4 hours ${ }^{[8]}$.

Experimental setup: The experimental setup used for the thermal diffusivity measurements ${ }^{[9]}$ consisted of the pyroelectric detector, a $52 \mu \mathrm{m}$ thick PVDF film attached to the samples with a thin layer of thermal grease. A thick vulcanized rubber layer was used as sample backing. The excitation source was a camera flash (Minolta 5400HS) with pulse duration of about 5 ms (that is much shorter than the pyroelectric response obtained from the samples) and of temporal shape that may be approximated with a finite square pulse. The signal from the PVDF transducer is recorded by a 400 $\mathrm{MHz}$ digital oscilloscope (LeCroy 9310A). The measurements were conducted at room temperature by averaging a total of 25 signals. The samples were polished to optimum measurement thickness of $\sim 1 \mathrm{~mm}$.

\section{RESULTS AND DISCUSSION}

Figure 1 shows PVDF response collected from the samples. As shown in the figures the model based on square pulse has reasonably good agreement with the experimental data with slight mismatches at the peak and the tail. The sources of the mismatch are from the heat loss that starts to dominate at the long time behavior at the tail and the deviation of the theoretical

Corresponding Author: M M Moksin, Department of physics, Faculty of Science, Universiti Putra Malaysia, 43400 UPM, Serdang, Selangor, MALAYSIA 

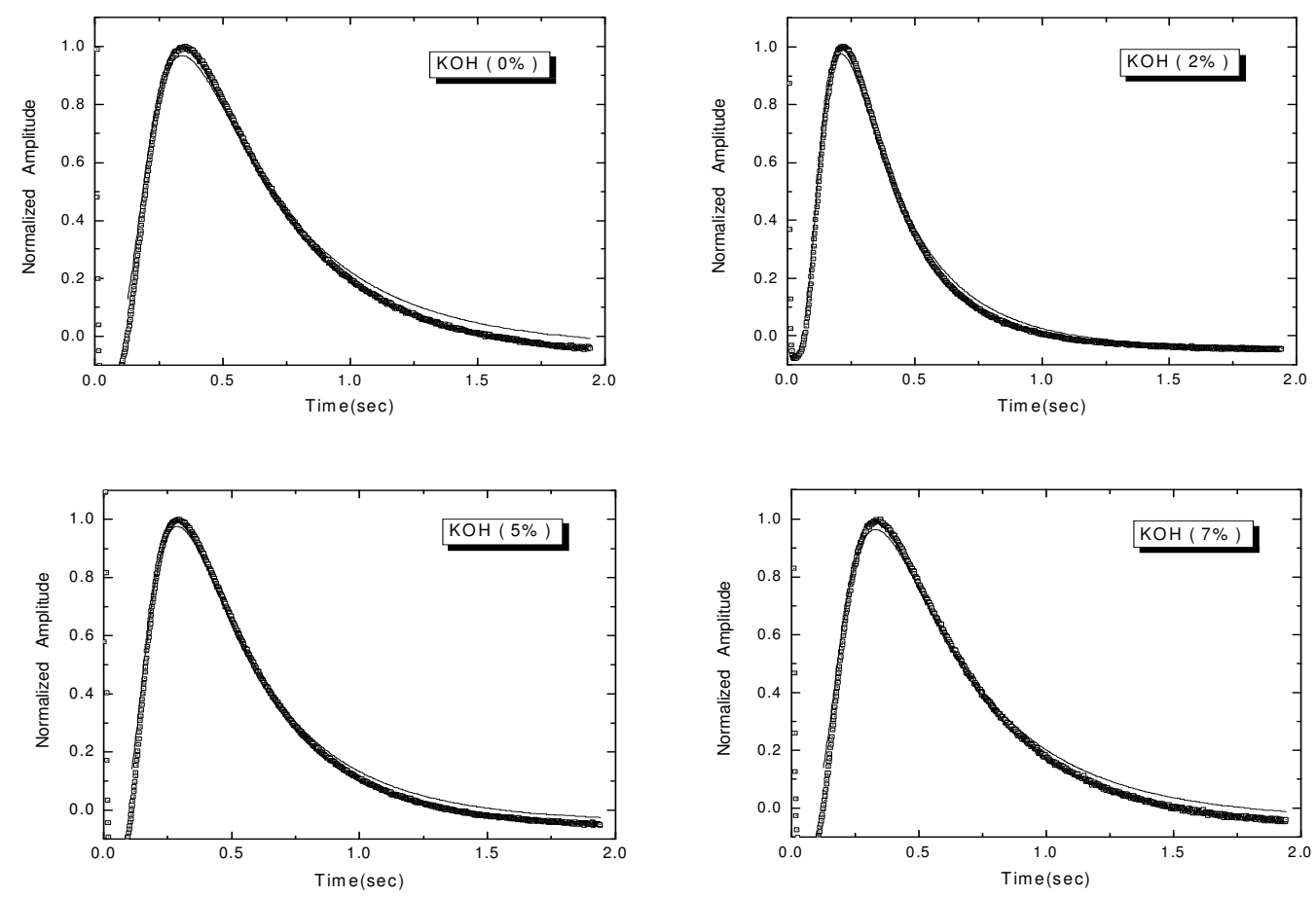

Fig. 1: Thermal wave response from carbon sample with different $\mathrm{KOH}(\%$ mole $)$ fitted with theoretical model (continuous line)

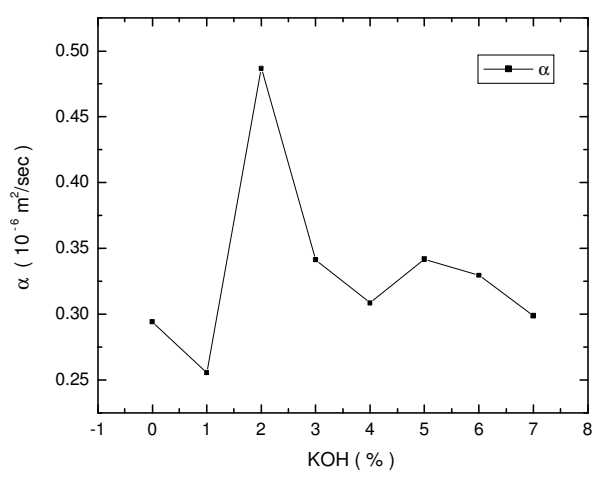

Fig 2: Thermal diffusivity as a function of $\mathrm{KOH}$ mole percent. The continuous line is for added clarity

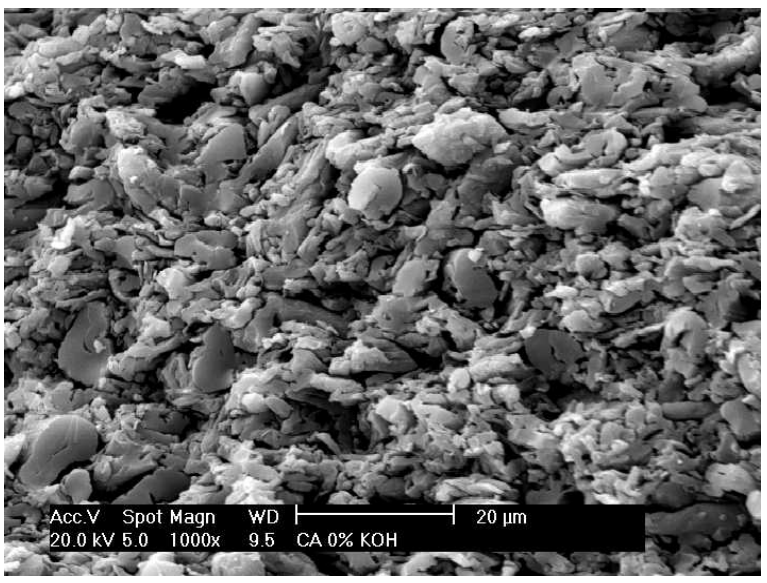

KOH (0\%) 


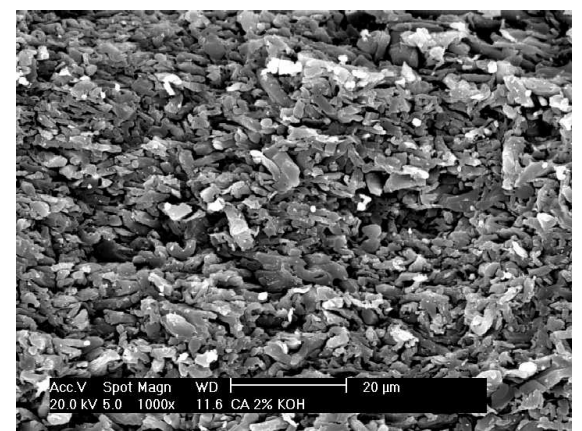

КОH (2\%)

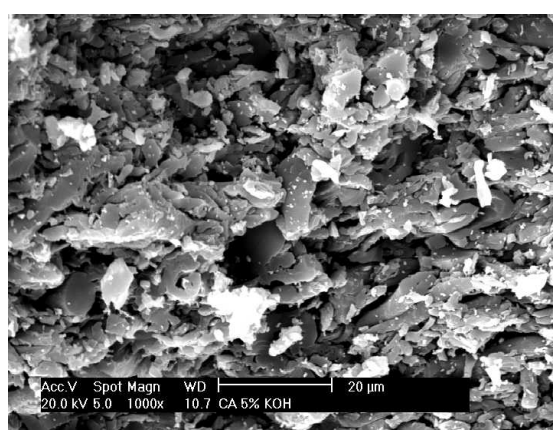

КОН (5\%)

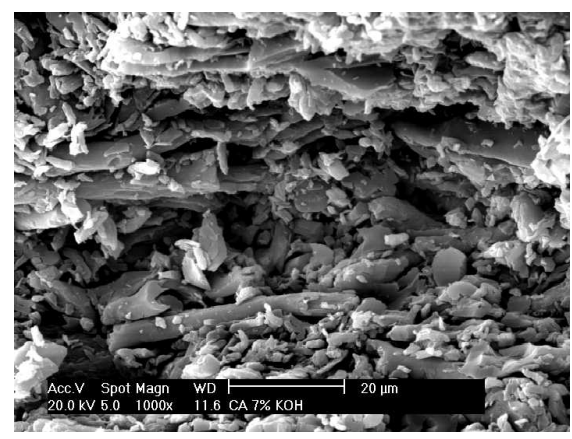

KOH (7\%)

Fig 3: SEM micrograph of carbon treated with different $\mathrm{KOH} \%$

square pulse from the actual experimental temporal profile of the camera flash. Therefore, the mismatch is less obvious in the data from the sample with higher thermal diffusivity as expected whereby heat loss is much lower when the signal is stronger and the temperature history is shorter. The thermal diffusivity for a set of carbon treated with $\mathrm{KOH}$ obtained from the curve fitting is summarized in figure 2 . The thermal diffusivity peaked itself at about $0.46 \times 10^{-6} \mathrm{~m}^{2} \mathrm{~s}^{-1}$ for the sample treated with $2 \%$ moles of $\mathrm{KOH}$. At other mole percentages the thermal diffusivity has slightly an upward trend towards higher mole percent.
The morphologies of the fractured surface for the $\mathrm{KOH}(0,2,5$ and $7 \%)$ treated carbon pellet are shown in Figure 3. The SEM micrograph shows that the grain size and inter-grain distance is the most distinguishable for the particular case of $\mathrm{KOH}(2 \%)$ treatment as compare to the rest which display almost similar features. Visually it is the most compact i.e. the least porous, smaller grain size and the best aligned among others. As evident in the Figure 2 the thermal diffusivity for the case of $\mathrm{KOH}(2 \%)$ is much higher than the others justifies better grain alignment and smaller porosity that aid to more efficient heat diffusion in the sample.

\section{CONCLUSION}

The positive effect of lower porosity and better grain alignment on the thermal diffusivity for a set of carbon pellets (CPs) treated with $\mathrm{KOH}$ has been described. This made thermal diffusivity value of $\mathrm{KOH}$ $(2 \%)$ higher than others.

\section{ACKNOWLEDGMENT}

We thank Malaysian Government and Universiti Putra Malaysia for research support through IRPA and Graduate Research Assistantship (M. Haydari).

\section{REFERENCES}

1. Kikuchi, T., Takahashi, T. and Nasu, S., 1972. Porosity dependence on thermal conductivity of uranium mononitride. Journal of Nuclear Materials. 4: 184-292

2. Takahashi, T. and Kikuchi, T., 1980. Porosity dependence on thermal diffusivity and thermal conductivity of lithium oxide $\mathrm{Li}_{2} \mathrm{O}$ from 200 to 900 ${ }^{\circ} \mathrm{C}$. Journal of Nuclear Materials. 91: 93-102

3. Shabbir, G., Maqsood, A. and Majid, C.A., 2000. Thermophysical properties of consolidated porous rocks. J.Phys.D: Appl:Phys. 33: 658-661

4. Grozescu, V.I., Moksin, M.M., Haydari, M., Yahaya, N. and Yunus, W.M.M., 2003. Application of the pyroelectric sensor for study porosity effect on the thermal diffusivity in Nickel Copper Zinc Ferrite. Proceeding of Asian Conference on Sensors: 65-68 
5. Bertolotti, M., Fabbri, L. and Sibilia, C. ,1989. The influence of porosity on the thermal diffusivity of gamma-aluminate ceramics. Material Science and Engineering. A109: 153-155

6. Haydari, M., Moksin, M.M., Yunus, W.M.M., Grozescu, V.I., Hamadneh, I. and Halim, S.A., 2004. Sintering time effect on thermal diffusivity in BSSCO doped with $\mathrm{Sm}$ at low temperature as revealed by flash method. Proceedings of the SPIE, 5581: 315-322

7. Haydari, M.Moksin, M.M., Yunus, W.M.M. and Grozescu, V. I., 2003. Theoretical model for thermal diffusivity calculation of solid material based on PVDF detection of signal generated by the square heating pulse temporal profile. Proceeding of Advance Technology Congress (Conference on Advance Materials). 36-44
8. Deraman, M., and Mohd Pauzi, I., 1995. Effect of particle size on the young modulus of carbon from oil palm bunches. Pertanika J. of Sci. \& Tech., 3(2): 203-209

9. Haydari, M., 2004. Development and applications of photoflash-PVDF technique in thermal diffusivity measurement at low temperatures. Ph.D. Thesis Universiti Putra Malaysia 